\title{
ESTIMATION OF TRANSMISSION RATE DEPENDENCE IN WI-FI NETWORK FROM INFORMATION CHARACTER
}

\author{
Bogdan Gumenyuk ${ }^{1)}$, Grygoriy Vaskiv ${ }^{2)}$, Volodymyr Yankevych ${ }^{3)}$ \\ 1) Lviv Polytechnic National University, 2, Profesorska Str., Lviv, 79013, Ukraine, bodia_g@list.ru \\ 2) Lviv Polytechnic National University, 2, Profesorska Str., Lviv, 79013, Ukraine, grygoriy_vaskiv@ukr.net \\ ${ }^{3)}$ Lviv Polytechnic National University, 2, Profesorska Str., Lviv, 79013, Ukraine, yankevych@gmail.com
}

\begin{abstract}
This paper shows the results of research in to data transmission rate in Wi-Fi networks from influence the topology of the network, information character, services of enciphering and authentification.
\end{abstract}

Keywords: Ad-hoc, 802.11b, 802.11g, Wi-Fi, Infrastructure mode, Interferences, Access point.

\section{INTRODUCTION}

With the modern implementation of new standards for high-rate Wi-Fi networks, mobile users are promised the levels of performance, data transmission rate (DTR), security and availability equivalent to those of traditional wired Ethernet. Such technologies are considered to be used for creating mobile and fixed wireless local networks and as a mean of comfortable accesses to the Internet.

However, Ethernet technology has developed and settled over a long time and it is regarded as the most popular LAN technology in today use. Now a Wi-Fi network is admired because it strikes a good balance between speed, cost and simple installation. But some business and home users are reluctant to migrate from their existing, wired LANs because they are confused over the abilities of wireless network.

This paper shows investigations in Wi-Fi networks, at their current abilities and their potential for becoming a mainstream connectivity solution. The investigation concentrates on IEEE $802.11 \mathrm{~b}$ and IEEE $802.11 \mathrm{~g}$ standards.

The wireless networks differ by their flexible architecture. But, although co-operation between Wi-Fi devices is described by protocols, there are interferences and various collisions in the real wireless networks.

The topology of the network has a substantial influence on data transmission rate. Because of simple connection to Wi-Fi network, there is a necessity in enciphering or in application of other protective measures to increase the data safety which substantially influences on fast-action.
Rate will also depend on the numbers of subscribers and on casual interferences which always are available. The casual interferences are caused by Wi-Fi devices, which are working in a nearby network by the operation of domestic and medical technique.

\section{THE REVIEW OF A SITUATION ON THE WIRELESS NETWORK}

Standards describe only two ways for creation wireless networks, there are Ad-hoc and Infrastructure modes. As they are similar on each other, also they have some differences are prior known before planning network.

You may to use Ad-hoc mode when it's necessary to make fast connection between several PC users (no more than five) for mobile network. If you use Ad-hoc mode the very important factor is that all computers must be against each others, because PC's position has huge influence on the date transmission rate. It is related to that transmitters power in wireless adapters is lower, than power of access point. According to that, in such kind of network working radius is half as much, than radius of network, which was built with the infrastructure mode.

Data transmission rate in network will be depended from standards which are used between wireless adapters. It's recommended to use adapters with same standards.

If a user is going to send a file to another used, data exchange rate with other PCs significantly decreases, that leads to certain delays in data transmission. It is also possible that several users 
would simultaneously exchange their files or download data from a single PC. In that case overall network throughput decreases rigidly. This is why it is recommended to limit number of connections down to 2-5 for proper network operation in Ad-hoc mode. If this number is bigger, it is recommended to switch access point to Infrastructure mode.

Access point is equipped with high-power radio, and this allows keeping link alive at greater distances. But this won't mean that bypassing such obstacles as walls, ceilings, trees, buildings etc becomes easy effort.

In case access point is being located inside a building, it should be placed between the PCs approximately at the center, to reach as many PCs within a direct sight as possible. This would allow users to operate in network with maximum data transfer rates and minimal interferences.

In case when access point is being located outdoors, clear line of sight between it and most distant network objects should be provided.

\section{AIMS AND TASKS}

The main goal of paper is research of influence various factors on the rate of data transmission in Wi-Fi networks and increasing efficiency of devices in such kind of networks. For that purpose specific work of different equipment was experimentally researched. Also proper software and standards of a wireless network for providing suitable service and access to the Internet was analyzed.

In addition, the aim of this paper is to provide detailed investigation and analysis of main performance issues related to wireless networks based on the IEEE $802.11 \mathrm{~b}$ and IEEE $802.11 \mathrm{~g}$ standards.

The main objective is to investigate wireless networks fast-acting from different topology and additional services.

\section{MAXIMUM DTR IN 802.11b/g STANDARDS}

A standard shows that maximum information transfer rate in protocol $802.11 \mathrm{~b}$ is $11 \mathrm{Mbps}$, and for protocol $802.11 \mathrm{~g}-54 \mathrm{Mbps}$.

However you must understand difference between maximum air rate and available date transmission rate. The point is that technology of access to the data transmission environment, structure of transmission frames, titles which are added to the transmission frames on the different levels of OSI model, and all that makes a huge block of service information. So, available or real date transmission rate is much lower than maximum air rate.

In particular, DTR depends on a wireless network structure. If all network clients use the same standards, for example $802.11 \mathrm{~g}$, a network is homogeneous and the DTR in such network is higher, than in the mixed network, where clients are both $802.11 \mathrm{~g}$ and $802.11 \mathrm{~b}$.

In addition, the DTR depends from used protocol (TCP or UDP) and from window size. The theoretical date transmission rates for the different types of networks and protocols are presented in Table 1.

Table 1. Transmission rate for different standards with $1.5 \mathrm{~Kb}$ window size

\begin{tabular}{|l|l|c|c|c|}
\hline Standards & $\begin{array}{l}\text { Modula- } \\
\text { tion }\end{array}$ & $\begin{array}{c}\text { Max. air } \\
\text { rate, } \\
\text { Mbps }\end{array}$ & $\begin{array}{c}\text { Theor. } \\
\text { available } \\
\text { DTR on } \\
\text { TCP, } \\
\text { Mbps }\end{array}$ & $\begin{array}{c}\text { Theor. } \\
\text { available } \\
\text { DTR on } \\
\text { UDP, } \\
\text { Mbps }\end{array}$ \\
\hline $802.11 \mathrm{~b}$ & CCK & 11 & 5,9 & 7,1 \\
\hline $\begin{array}{l}802.11 \mathrm{~g}(3 \\
802.11 \mathrm{~b})\end{array}$ & $\begin{array}{l}\text { OFDM/ } \\
\text { CCK }\end{array}$ & 54 & 14,4 & 19,5 \\
\hline $802.11 \mathrm{~g}$ & $\begin{array}{l}\text { OFDM/ } \\
\text { CCK }\end{array}$ & 54 & 24,4 & 30,5 \\
\hline
\end{tabular}

\section{EXPERIMENTAL SETTING}

Hardware. The all of experiments were carried out on Lviv Polytechnic National University at Institute of Telecommunications and Radioelectroncs. Current set-up consists of three desktop PCs connected via D-Link/AirPlus G PCI adapters into an ad-hoc and infrastructure mode wireless LAN. In addition, a two notebook computers with integrated Wi-Fi adapters were used. In particular, measuring was conducted with a spectrum analyzer Fs-300.

Measurement Software. There are a number of software tools currently available which could be utilized in order to carry out the required measurements.

Iperf is using a client server model to determine total throughput as well as measurements of delay and packet loss. It supports TCP window size and it runs tests by amount transferred or for a specified period of time. Iperf runs on the majority of popular OS platforms.

Netperf is a client/server based benchmark that can be used to measure various aspects of networking performance. It was developed by Hewlett Packard and it can be used on majority of network implementations. Its primary focus is on bulk data transfer and request/response performance using either TCP or UDP.

Total Commander (former Wincmd) is a file manager for Windows similar to the Windows Explorer. Total Commander also integrates an FTP 
client: hit control-F to connect to an FTP site, and it shows up in Total Commander just like a local drive or a network drive. Copying files between a laptop, a file server, an FTP server, or even between two FTP servers all work identically and, in particular, it shows date transmission rate when you are copying.

Environment The workstations are situated in a small room and are placed few meters apart. The room contains other hardware but there are no any direct sources of interference.

\section{EXPERIMENT CONDUCTIONS}

In the process of working on research project a few experiments were done. The goal of the first block of the experiments was two devices a notebook and personal computer connected a topology point to point (Ad-hoc) in the standard IEEE $802.11 \mathrm{~g}$ on the frequency $2,412 \mathrm{GHz}$ (1st channel). The experiment was carried out in the same place without substantial interferences on the band of frequency from $2,4 \mathrm{GHz}$ till $2,5 \mathrm{GHz}$, coming from the shows of spectrum analyzer FS-300 Fig.1.

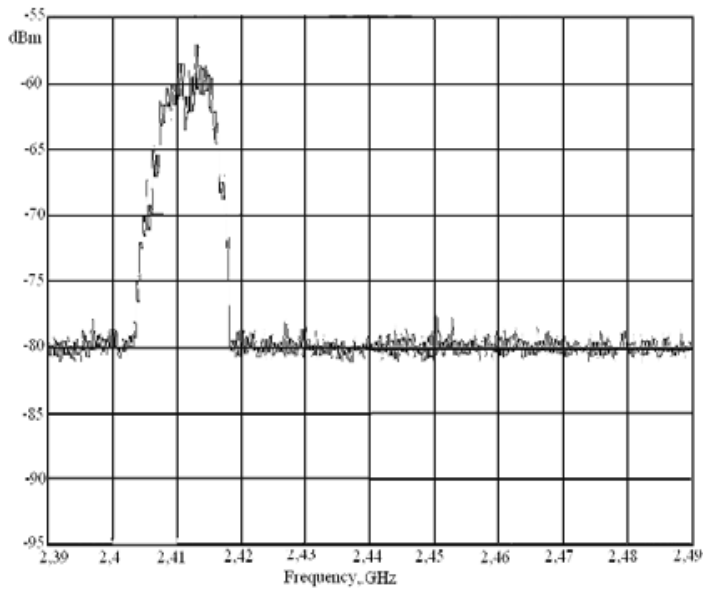

Fig. 1 - Frequency band from 2,4GHz till 2,5GHz without any substantial interferences.

In the process transfer of different test files, the average rate of data communication was gained within an interval $18,0-19,2 \mathrm{Mbps}$, but the maximum speed is $24,4 \mathrm{Mbps}$. Another experiment was conducted for the standard IEEE $802.11 \mathrm{~b}$ and we achieved value of data rate is in a range $5,00-5,23 \mathrm{Mbps}$, but the maximum speed is $5,9 \mathrm{Mbps}$. The same experiments were done again with authentification and enciphering. The results obtained in Table 2 .
Table 2. Results of the 1st block of experiments, Mbps

\begin{tabular}{|c|c|c|c|c|}
\hline $\begin{array}{c}\text { Inf. } \\
\text { charac- } \\
\text { ter }\end{array}$ & $\mathbf{8 0 2 . 1 1 b}$ & $\mathbf{8 0 2 . 1 1 g}$ & $\begin{array}{c}\mathbf{8 0 2 . 1 1 b} \\
\mathbf{8} \\
\text { encip- } \\
\text { hering }\end{array}$ & $\begin{array}{c}\mathbf{8 0 2 . 1 1 g} \\
\mathbf{8} \\
\text { encip- } \\
\text { hering }\end{array}$ \\
\hline File 1 & 5,23 & 19,2 & 4,97 & 17,67 \\
\hline File 2 & 5,19 & 18,5 & 4,93 & 17,37 \\
\hline File 3 & 5,09 & 18,05 & 4,64 & 16,38 \\
\hline
\end{tabular}

In the second block of experiments we built a network by an infrastructural method (base station and two points) using one more leptop. We made the same kinds of experiments as in first block. The results obtained you can see in Table 3 .

Table 3. Results of the 2nd block of experiments, Mbps

\begin{tabular}{|c|c|c|c|c|}
\hline $\begin{array}{c}\text { Inf. } \\
\text { charac- } \\
\text { ter }\end{array}$ & $\mathbf{8 0 2 . 1 1 b}$ & $\mathbf{8 0 2 . 1 1 g}$ & $\begin{array}{c}\mathbf{8 0 2 . 1 1 b} \\
\mathbf{8} \\
\text { encip- } \\
\text { hering }\end{array}$ & $\begin{array}{c}\mathbf{8 0 2 . 1 1 g} \\
\mathbf{8} \\
\text { encip- } \\
\text { hering }\end{array}$ \\
\hline File 1 & 4,67 & 18,7 & 4,39 & 17,42 \\
\hline File 2 & 4,5 & 18,2 & 4,21 & 17,14 \\
\hline File 3 & 4,02 & 17,62 & 3,81 & 16,23 \\
\hline
\end{tabular}

In the third block of the experiments we built a network by two previous methods of network construction with the using more personal computers and leptop. The results obtained you can see in Table 4.

Table 4. Results of the 3rd block of experiments, Mbps

\begin{tabular}{|c|c|c|c|c|}
\hline $\begin{array}{c}\text { Inf. } \\
\text { charac- } \\
\text { ter }\end{array}$ & $\mathbf{8 0 2 . 1 1 b}$ & $\mathbf{8 0 2 . 1 1 g}$ & $\begin{array}{c}\mathbf{8 0 2 . 1 1 b} \\
\mathbf{8} \\
\text { encip- } \\
\text { hering }\end{array}$ & $\begin{array}{c}\mathbf{8 0 2 . 1 1 g} \\
\mathbf{8} \\
\text { encip- } \\
\text { hering }\end{array}$ \\
\hline File 1 & 3,71 & 13,77 & 3,49 & 12,71 \\
\hline File 2 & 3,53 & 12,73 & 3,35 & 11,76 \\
\hline File 3 & 3,31 & 12,65 & 3,12 & 11,34 \\
\hline
\end{tabular}

\section{CONCLUSION}

According to the results of experiments it shows that a data transmission rate depends the topology of the network within the limits of $20-35 \%$, using ciphering services and authentification in a range $7-10 \%$, and information character is described in an interval $8-16 \%$. It is necessary to point out that the additional supervision dependence of fast-action work of wireless network, that at the use of the operating system Linux results of experiments are on a range $12-15 \%$ higher, than at OS of Windows.

In particular, using physical interferences and services slows the rate of data transmission. 


\section{REFERENCES}

[1] P. Roshan, J. Leary. 802.11 Wireless LAN fundamentals. Cisco, Indianapolis, Ind., 2003.

[2] R. Flickenger. Building Wireless Community Networks. O'Reilly, US, 2003.

[3] E. Geier. Wi-Fi Hotspots. Cisco Press, US, 2006 focusing on 802.11 IEEE standards family, and providing researches in corresponding areas.

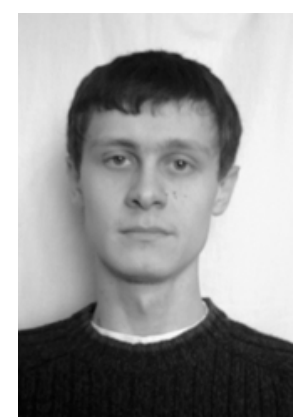

Bogdan Gumenyuk, born in 1985 in Lviv, Ukraine. With high interest in modern technologies, especial communication and computer science, in 2002 he entered Lviv Polytechnic National University, where he was studying at Institute of Telecommunications and Radioelectroncs till winter 2007 as student. After successfully graduation there, he made decision to continue science work as a post-graduated student. Currently Bogdan is a 1st year post-graduate student at "Lviv polytechnic", still greatly interested in modern communication technologies, focusing on 802.11 IEEE standards family, and providing researches in date transmission rate.

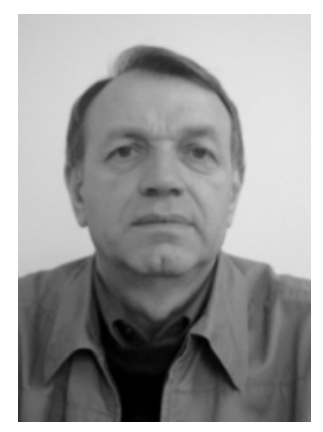

Grygoriy Vaskiv, born in 1954. $\mathrm{He}$ is Associate Professor in Lviv Polytechnic National University at Institute of Telecommunications and Radioelectroncs. From 1975 to 1980 has been studying at Lviv's Polytechnic Institute. From 1981 to 1983 has been studying as post-graduate student at same Institute. In 1989 has protected candidate dissertation in the Kaunas city, Lithuania. His main research interests are code assignment in wireless and cellular networks, protocols for pricing and Quality of Service in wireless networks.

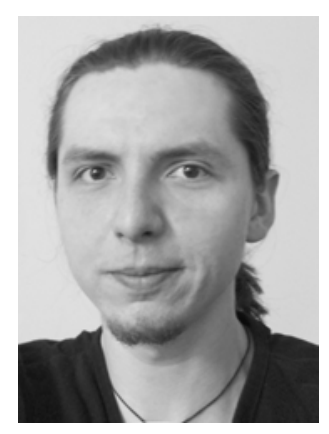

Volodymyr Yankevych, born in 1983 in Stryi (Lviv region, Ukraine). Due to deep interest in electronics and modern technologies, in 2000 he entered Lviv Polytechnic National University, where he was studying at Institute of Telecommunications and Radioelectroncs till autumn 2005 as student. After successfully to obtaining a distinction, there was decision made to continue science and write a dissertation concerning wireless communications there. Currently Volodymyr is a 3rd year postgraduate student at "Lviv polytechnic", still greatly interested in modern communication technologies, 